\title{
A Protocol for the Evaluation of Nutritional and Functional Status Evolution During a Multidisciplinary Rehabilitation Program for Patients after SARS-CoV-2 Pneumonia ${ }^{\dagger}$
}

\author{
Diogo Sousa-Catita ${ }^{1,2, * \mathbb{C}}$, Catarina Godinho ${ }^{1} \mathbb{1}$ and Jorge Fonseca ${ }^{1,3}$ \\ 1 PaMNEC-Grupo de Patologia Médica, Nutrição e Exercício Clínico, CiiEM-Centro de Investigação \\ Interdisciplinar Egas Moniz, 2829-511 Almada, Portugal; cgodinho@egasmoniz.edu.pt (C.G.); \\ jorgedafonseca@hotmail.com (J.F.)

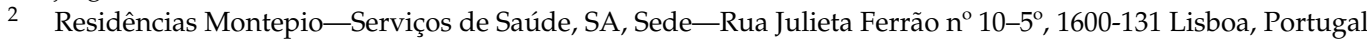 \\ 3 GENE-Artificial Feeding Team, Gastroenterology Department, Hospital Garcia de Orta, \\ 2805-267 Almada, Portugal \\ * Correspondence: diogo.rsc2@gmail.com \\ + Presented at the 5th International Congress of CiiEM-Reducing Inequalities in Health and Society, Online, \\ 16-18 June 2021
}

Citation: Sousa-Catita, D.; Godinho, C.; Fonseca, J. A Protocol for the Evaluation of Nutritional and Functional Status Evolution During a Multidisciplinary Rehabilitation Program for Patients after SARS-CoV-2 Pneumonia. Med. Sci. Forum 2021, 5, 16. https://doi.org/ $10.3390 / \mathrm{msf} 2021005016$

Academic Editors: Helena Barroso and Cidália Castro

Published: 20 July 2021

Publisher's Note: MDPI stays neutral with regard to jurisdictional claims in published maps and institutional affiliations.

Copyright: (c) 2021 by the authors. Licensee MDPI, Basel, Switzerland. This article is an open access article distributed under the terms and conditions of the Creative Commons Attribution (CC BY) license (https:// creativecommons.org/licenses/by/ $4.0 /)$.

\begin{abstract}
Nutrition status is a major issue of the COVID-19 pandemic. Many factors associated with worse prognosis risk are related to nutrition. Patients received after hospital discharge for pneumonia due to SARS-Cov-2 were submitted to a multidisciplinary rehabilitation program. This study aimed to analyze the nutritional and functional status after SARS-Cov-2 pneumonia and evaluate the impact of a multidisciplinary rehabilitation program.
\end{abstract}

Keywords: SARS-CoV-2 pneumonia; nutritional and functional status; rehabilitation program

\section{Introduction}

Proper nutrition and good nutritional status have been gaining importance in the context of the COVID-19 pandemic. Food and nutrition are playing an important role in the prevention of disease and as one of the areas of priority intervention to minimize the consequences of this viral infection. Having a balanced diet contributes to a better nutritional status, an adequate immune response, decreases the risk of severe evolution and complications. Although the entire population is susceptible to SARS-CoV-2, most hospitalized patients are elderly and/or with nutrition-related chronic diseases (obesity, diabetes, hypertension, and cardiovascular diseases) [1]. All of these have the worst nutritional status, compromised immune system, and weakened respiratory system. The factors that have been associated with an increased risk of developing the severe COVID-19 disease and worse prognosis, such as hypoalbuminemia, lymphopenia, sarcopenia/fragility, high body mass index (BMI), and obesity, are related with malnutritional status [2]. Therefore, nutritional status plays an important role in COVID-19 being associated with a greater risk of developing severe illness and with a greater risk of hospitalization, ICU stay and death. On the other hand, patients who recovered from SARS-Cov-2 pneumonia present a decline in nutritional status, caused by the catabolic effect of the disease and by the hospital stay, with loss of muscle mass and function.

This study aimed to evaluate: (1) the nutritional and functional status of patients when they are received at the Residências Montepio (RM), transferred from hospitals after SARS-Cov-2 pneumonia; (2) the nutritional and functional status at the end of the rehabilitation program; and (3) the impact on the nutritional and functional status of the integrated and multidisciplinary rehabilitation program carried out after SARS-Cov-2 pneumonia. In addition, we intend to (4) assess food intake during the first $48 \mathrm{~h}$ of the 
rehabilitation program, because we suspect, from previous experience with these patients, that oral intake can be significantly reduced when compared with nutritional needs.

\section{Materials and Methods}

This is an observational, analytical, longitudinal study, evaluating no less than 110 adults. This will not have a pre-established duration, but it should not exceed 6 months. Statistical analysis will be used by SPSS to compare and correlate the nutritional and functional status variables, to see the nutritional and function impact of multidisciplinary rehabilitation program. The inclusion criteria are patients hospitalized in RM with a history of SARS-Cov-2 pneumonia agreeing to participate in the study and signing the informed consent. The exclusion criteria are patients with severe chronic underlying disorders that may clearly and very probably have a negative impact on the rehabilitation program. Each patient will only be part of the study while they are hospitalized in RM, from entrance (T0) until discharge (T2) 30 days later. At RM, they are submitted to an integrated and multidisciplinary rehabilitation program including 30 days of intensive rehabilitation, with physical therapy, occupational therapy, psychological intervention, speech therapy and personalized nutrition care, associated with medical and nursing care.

Each patient will be evaluated at three moments:

T0-Upon admission to the unit

T1-after 15 days

T2-Within $48 \mathrm{~h}$ before discharge

Each assessment will include the following parameters:

- Anthropometric data: weight, height, body mass index (BMI) according to age, geminal perimeter, brachial perimeter, tricipital skinfold (TSF), calculation of the muscular circumference of the arm.

- Functional assessment: hand grip strength assessed with pressure dynamometry.

- Scales: MNA [3]; GLIM and assessment of food intake in the first $24 \mathrm{~h}(24 \mathrm{~h}$ food recall) [4].

- Laboratory: routine blood count and serum albumin.

\section{Results and Discussion}

The present protocol was ratified by the Scientific Council of the Egas Moniz Higher School of Health and approved by the Ethics Committee (\# 909). By 25 April, 107 patients aged 42-90 years were enrolled and 83 had already finished their protocols. We hope this protocol will show us if the different nutritional and function status in SARS-Cov-2 pneumonia patients has an influence on their recovery and future.

Institutional Review Board Statement: The study was conducted according to the guidelines of the Declaration of Helsinki, and approved by the Ethics Committee of Egas Moniz Higher School of Health (protocol code \#909 of 17 December 2020).

Informed Consent Statement: Informed consent was obtained from all subjects involved in the study.

Data Availability Statement: Not applicable.

Acknowledgments: The authors declare no acknowledgments.

Conflicts of Interest: The authors declare no conflict of interest.

\section{References}

1. Aggarwal, S.; Garcia-Telles, N.; Aggarwal, G.; Lavie, C.; Lippi, G.; Henry, B.M. Clinical features, laboratory characteristics, and outcomes of patients hospitalized with coronavirus disease 2019 (COVID-19): Early report from the United States. Diagnosis 2020, 7, 91-96. [CrossRef] [PubMed]

2. Arentz, M.; Yim, E.; Klaff, L.; Lokhandwala, S.; Riedo, F.X.; Chong, M.; Lee, M. Characteristics and Outcomes of 21 Critically Ill Patients with COVID19 in Washington State. JAMA 2020, 323, 1612-1614. [CrossRef] [PubMed] 
3. Kaiser, M.J.; Bauer, J.M.; Ramsch, C.; Uter, W.; Guigoz, Y.; Cederholm, T.; Thomas, D.R.; Anthony, P.; Charlton, K.E.; Maggio, M.; et al. Validation of the Mini Nutritional Assessment short-form (MNA $\left.{ }^{\circledR}-S F\right)$ : A practical tool for identification of nutritional status. J. Nutr. Health Aging 2009, 13, 782-788. [CrossRef]

4. Questionnaires from Nutrition Day (Sheet 2). Available online: https://www.nutritionday.org/cms/front_content.php?idart=477 (accessed on 11 December 2020). 